\title{
Roles of the bias fields in the exchange interaction between the electron and hole spins in quantum wells
}

\author{
M. Idrish Miah ${ }^{1,2, *}$ \\ ${ }^{1}$ Nanoscale Science and Technology Centre and School of Biomolecular and Physical \\ Sciences, Griffith University, Nathan, Brisbane, QLD 4111, Australia. \\ ${ }^{2}$ Department of Physics, University of Chittagong, Chittagong, Chittagong - 4331, \\ Bangladesh.
}

We study the electric-field effects on the spin polarization (P) in dependence of the strength and orientation of the magnetic field in GaAs quantum wells by photoluminescence measurements. The $P$ in a transverse magnetic field is found to oscillate. The transverse electron and heavy-hole $g$-factors are estimated from the dependences of the oscillation frequency on magnetic field and applied bias. Measurements with the angular variations of the magnetic field show that both the oscillation frequency and decay rate are increased with increasing the angle from the transverse direction. It is, however, shown that the application of the bias to the quantum wells weakens the exchange interaction between the electron and hole spins.

\footnotetext{
*Electronic mail: m.miah@griffith.edu.au
} 
Spin electronics [1] or spintronics [2], or spin physics in a broader sense [3,4], is a promising field of research whereby the spin degree of freedom in electronic devices is exercised. Recently, it has gained a lot of attention [2,4-7], which may result in devices with increased capability beyond well-established data storage applications.

One of the requirements necessary in developing spintronic devices is the efficient generation of spins in a semiconductor and transporting them reliably (without loss of the spin polarization) over reasonable distances, i.e. distances that are comparable to the device dimensions. Generation of spin polarization can be achieved either by optical methods or by injection from the magnetic semiconductors, or ferromagnetic contacts $[2,6]$. However, the spin generation by the optical methods has been successful and the high spin-polarization of electrons in semiconductor heterostructures has been obtained [2]. Despite substantial progress in optical spin generation, a further task is to investigate the spin dynamics in heterostructures.

In the present investigation, we focus on spin dynamics in quantum wells (QWs) by circularly polarized photoluminescence (PL) measurements. The PL circular polarization $(P)$ in a transverse magnetic field is found to oscillate. The oscillation frequency and decay rate as a function of the bias and magnetic field are obtained, and the transverse components of the electron and heavy-hole $g$-factors are estimated. The $P$ with variations of the magnetic field from the transverse direction is also studied.

Samples were GaAs double QWs (8 $\mathrm{nm}$ thick) separated by a relatively thin ( $20 \mathrm{~nm})$ $\mathrm{Al}_{0.3} \mathrm{Ga}_{0.7} \mathrm{As}$ barrier. The sample was mounted in a chip-carrier. We measured the PL excited by circularly polarized ps pulses of a tunable Ti:sapphire laser with a 
repetition rate of $76 \mathrm{MHz}$ using a streak camera. A scheme illustrating the experimental setup is shown in Fig. 1 . All the measurements were done at $4.2 \mathrm{~K}$. The orientation of the magnetic field with respect to the growth axis of the heterostructure was changed by rotating the chip-carrier. The polarization of the exciting beam was converted from linear to circular polarization by a quarter wave plate.

The $P$ in QWs was studied in dependence of the strength and orientation of the magnetic field. The $P$ was calculated using the relation $P=\left(I_{++}-I_{+-}\right) /\left(I_{++}+I_{+-}\right)$, where $I_{++}\left(I_{+-}\right)$is the intensity of $\mathrm{PL}$ in the $\mathrm{R}(\mathrm{L})$ circularly polarization under $\mathrm{R}$ circularly polarized excitation. In Fig. 2, we plot $P$ in the absence of a bias and for 5-T transverse magnetic field. As can be seen, the $P$ oscillates, but it decays rapidly with time. In the presence of the applied bias, the electric field reduces the electron-hole exchange coupling by spatially separating the charges, and as a result, the interaction of the hole spin with phonons becomes stronger than the exchange interaction [8]. This leads to breakage of the coupling between the electron and hole spins.

In the presence of the $-2.5 \mathrm{~V}$ bias, the $P$ for 2 and $5 \mathrm{~T}$ transverse magnetic fields are shown in Figs. 3 and 4 . As can be seen, the $P$ shows distinct oscillations symmetric with respect to the horizontal axis. An analysis of the polarization dynamics in the transverse magnetic field gives an additional evidence for the exchange interaction suppression in an external electric field. In the absence of the bias, there involves only the behaviour of the exciton spin as a whole. The magnetic field mixes the excitonic states, and as a result, the $\mathrm{R}$ circularly polarized light becomes capable of exciting several states. The dynamics of the polarized PL is controlled here by the interference of the states of the exciton fine structure split by the combined action of the magnetic 
field and exchange coupling governed by the external electric field [9,10]. As the Fig. 2 demonstrates, the $P$ varies in time in a rather complicated fashion of the PL polarization kinetics, reflecting superposition of the beats at several frequencies. In the presence of the bias, the hole spin rapidly relaxes and thus the electronic spin freely precesses around the magnetic field orientation. The application of the bias to the samples weakens the exchange interaction between the electron and hole spins.

The projection of the electron spin onto the direction of observation oscillates in time with the Larmor frequency

$\omega=g \mu_{B} H / \hbar$,

where $H$ is the magnetic field, $\mu_{\mathrm{B}}$ is the Bohr magneton and $g$ is the electron- $g$ factor. The $P$ should oscillate between $+1(100 \%)$ and $-1(-100 \%)$ in the absence of decay with the same frequency.

The data presented in Figs. 2-4 are fitted (solid lines) by the exponentially damping harmonic function [11] $P=P_{0} \exp (-t / \tau) \cos \omega t$, where $P_{0}$ is the initial amplitude and $\tau$ is the decay time. From the fits to the experimental data, we have obtained the values of $\omega$ and $\tau$. The dependences of $\omega$ on the applied bias and magnetic field are shown in Fig. 5. As can be seen, $\omega$ increases linearly with increasing magnetic field and decreases with the negative applied bias. The lines in Fig. 5 are fits made by Eq. (1). The slopes of the fits give the values of the transverse electron $g$-factor $\left(g_{\perp}\right)$ for zero and $-2.5 \mathrm{~V}$ biases. The obtained values are $g_{\perp}=6.2 \times 10^{-2}$ and $20.8 \times 10^{-2}$ for zero 
and $-2.5 \mathrm{~V}$ biases respectively. The value of $g_{\perp}$ for the zero bias corresponds to the heavy-hole $g$-factor. The values agree with those obtained in Refs. [12,13].

The decay rate, estimated at $-2.5 \mathrm{~V}$, as a function of the transverse magnetic field is shown in Fig. 6. As can be seen, the decay rates are within a few per ns for up to $9 \mathrm{~T}$ and increases with increasing the strength of the magnetic field. The observed $P$ decay rates correspond to electron spin dephasing rates in the samples for transverse magnetic fields and are in an agreement with those obtained in other observations [2].

Variations of $P$ with the angle between the magnetic field and the transverse direction are shown in Fig. 7 . When the angle $\theta$ deviates from $90^{\circ}$, a non-oscillating weakly damping component arises and its amplitude and the amplitude of the oscillating component increase with the deviation angle. From Fig. 7, one can also see that the deviation from the Voigt configuration $\left(\theta=90^{\circ}\right)$ is accompanied by an increase in the oscillation frequency. The increase in $\omega$ upon deviation of the magnetic field from the transverse direction is related to the anisotropy of the electron $g$-factor resulting from the quantum size effect or quantum confined (quantization) effect. The splitting between the spin sublevels of the free electron in QWs is given [14] by $\Delta^{\sigma}=\mu_{B} H\left\{\left(g_{\square} \cos \theta\right)^{2}+\left(g_{\perp} \sin \theta\right)^{2}\right\}^{1 / 2}$, where $g_{\square}$ and $g_{\perp}$ are the longitudinal and transverse components of the electron $g$-factor respectively. As can also be seen, the change of $\theta$ from $90^{\circ}$ is accompanied by an increase in the decay rate. Since $g_{\square}$ and $g_{\perp}$ are close in magnitude, contribution from a difference of their values is not dominant. Therefore, the angular dependence of the decay of the oscillations is mainly related to the residual exchange interaction between the electron spin and rapidly relaxing spin of the hole [15]. 
In summary, the electric-field effects on the optically induced spin polarization in GaAs quantum wells were investigated by PL measurements. In a transverse magnetic field, the oscillation frequency and decay rate of $P$ were found to vary with the strengths of the magnetic field and applied bias. From their dependences, the transverse components of the electron and heavy-hole $g$-factors were estimated. The $P$ with variations of the magnetic field from the transverse direction was also studied and their dependences were discussed. 


\section{References}

[1] Spin Electronics, M. Ziese and M. J. Thornton, Eds., Vol. 569, SpringerVerlag, Heidelberg, 2001.

[2] Semiconductor Spintronics and Quantum Computation, D.D. Awschalom, D. Loss, and N. Samarth, Eds., Springer, Berlin, 2002.

[3] M. I. Dyakonov and V. I. Perel, Sov. Phys. JETP 33, 1053 (1971).

[4] M. I. Dyakonov and A. V. Khaetskii, Spin Hall Effect (Spin Physics in Semiconductors, M. I. Dyakonov, Ed., Springer-Verlag, Berlin, 2008).

[5] S. D. Sarma, Am. Sci. 89, 516 (2001).

[6] M. I. Miah, J. Optoelectron. Adv. Mater. 10, 2487 (2008).

[7] G. A. Prinz, Science 282, 1660 (1998).

[8] A. G. Mal’shukov, K. A. Chao and M. Willander, Phys. Rev. B 52, 5233 (1995)

[9] M. Dyakonov, X. Marie, T. Amand, P. Le Jeune, D. Robart, M. Brousseau, and J. Barrau, Phys. Rev. B 56, 10412 (1997).

[10] V. K. Kalevich, B. P. Zakharchchenya, K. V. Kavokin, A. V. Petrov, P. Le Jeune, X. Marie, D. Robart, T. Amand, J. Barrau, and M. Brouseau, Phys. Solid State 39, 681 (1997).

[11] F. Mandl, Quantum Mechanics, John Wiley \& Sons, New York, 1998.

[12] E. L. Ivchenko and A. A. Kiselev, Sov. Phys. Semicond. 26, 827 (1992).

[13] A. Malinowski and R. T. Harley, Phys. Rev. B 62, 2051 (2000).

[14] H. W. van Kesteren, E. C. Cosman, W. A. J. A. van der Poel, and C. T. Foxon, Phys. Rev. B 41, 5283 (1990).

[15] G. E. Pikus and A. N. Titkov, In Optical Orientation (Modern Problems in Condensed Matter Science, Vol. 8, North-Holland, Amsterdam, 1984). 


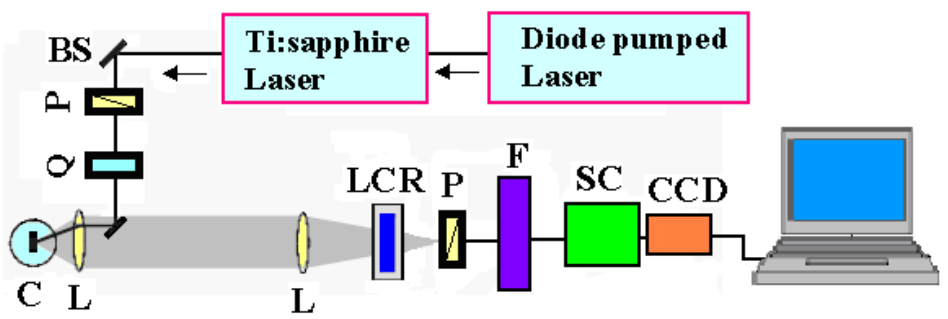

Fig. 1. A scheme illustrating the experimental setup (BS: Beam splitter; P: Polarizer; Q: Quarter wave plate; L: Lens; LCR: Liquid crystal retarder; F: Filter; SC: Streak camera). The sample was placed in a LH temperature-regulated cryostat (C). 


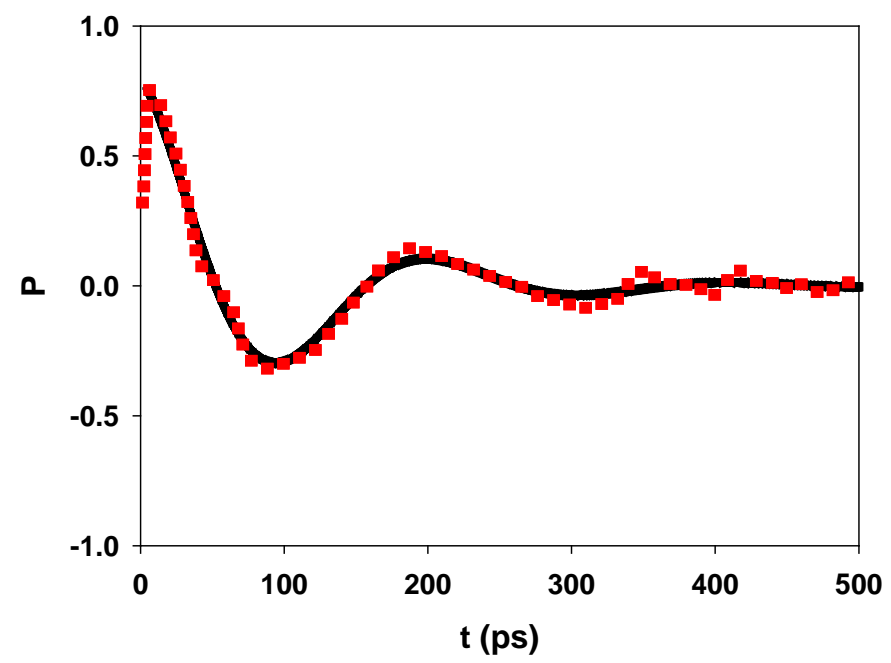

Fig. 2. The $P$ in the presence of the transverse magnetic field of $5 \mathrm{~T}$ and for zero bias. 


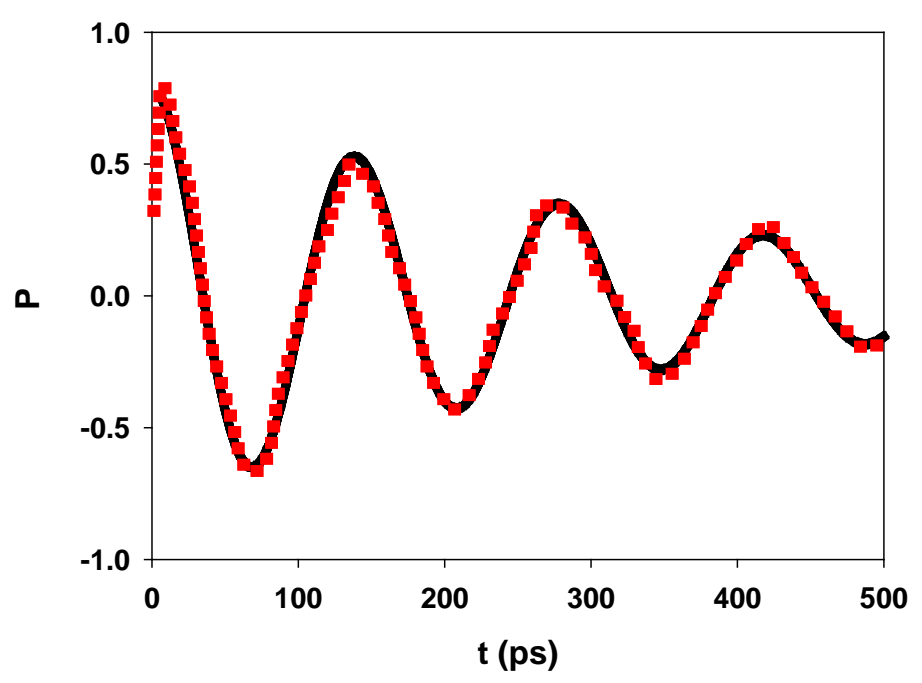

Fig. 3. The $P$ in the presence of a bias $(-2.5 \mathrm{~V})$ and the transverse magnetic field of 2 T. 


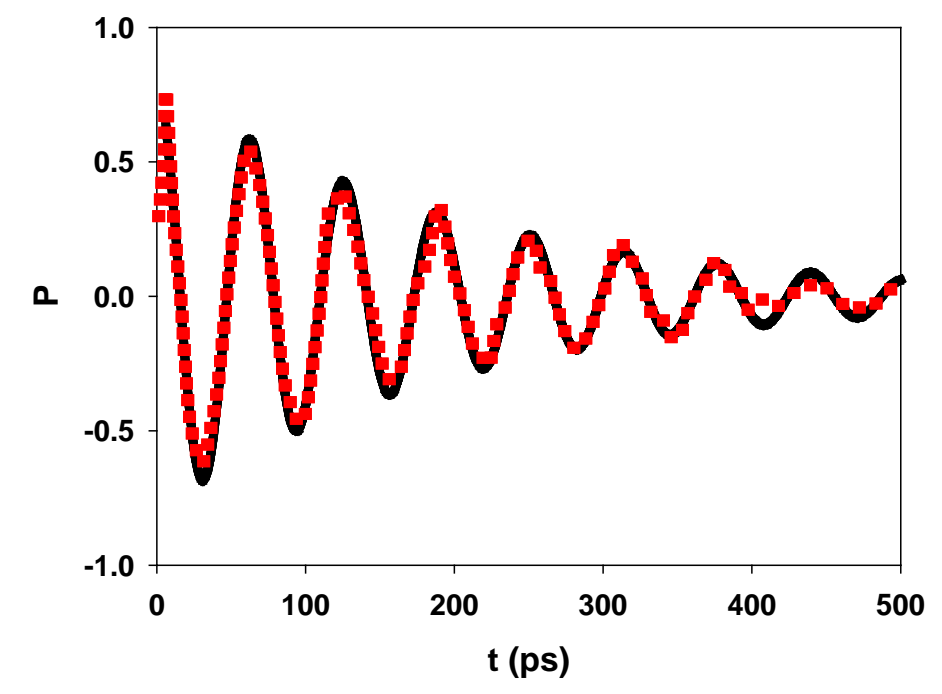

Fig. 4. The $P$ in the presence of a bias $(-2.5 \mathrm{~V})$ and the transverse magnetic field of 5 T. 


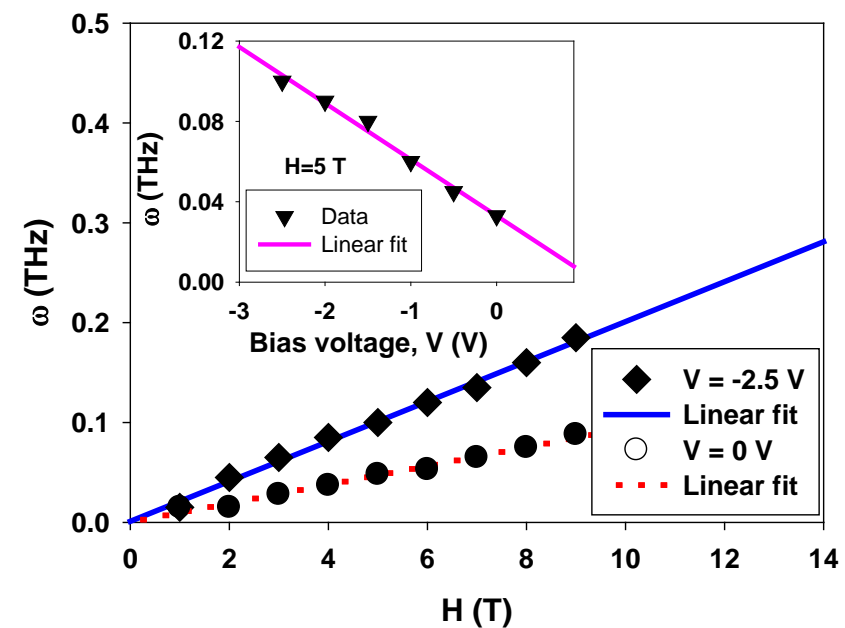

Fig. 5. Dependences of the oscillation frequency on the transverse magnetic field and applied bias (insert). 


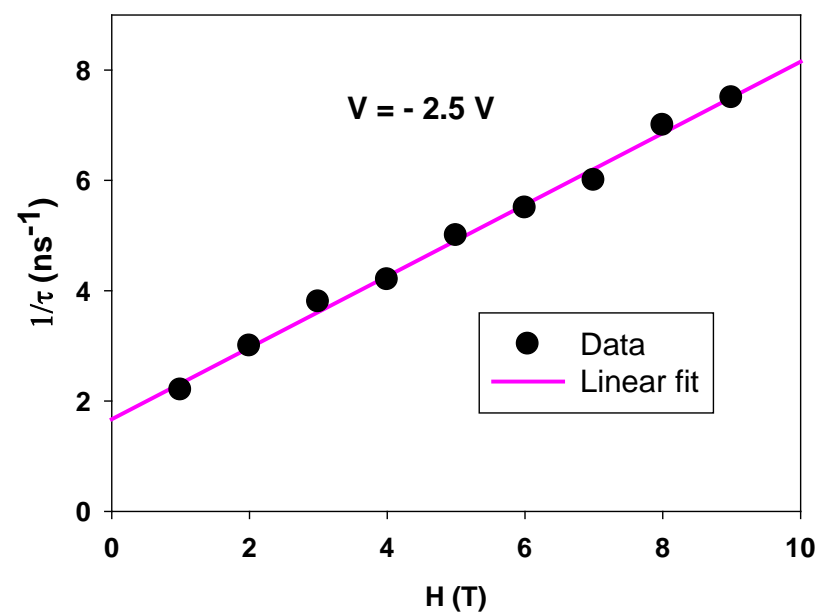

Fig. 6. Variations of the decay rate with the transverse magnetic field. 


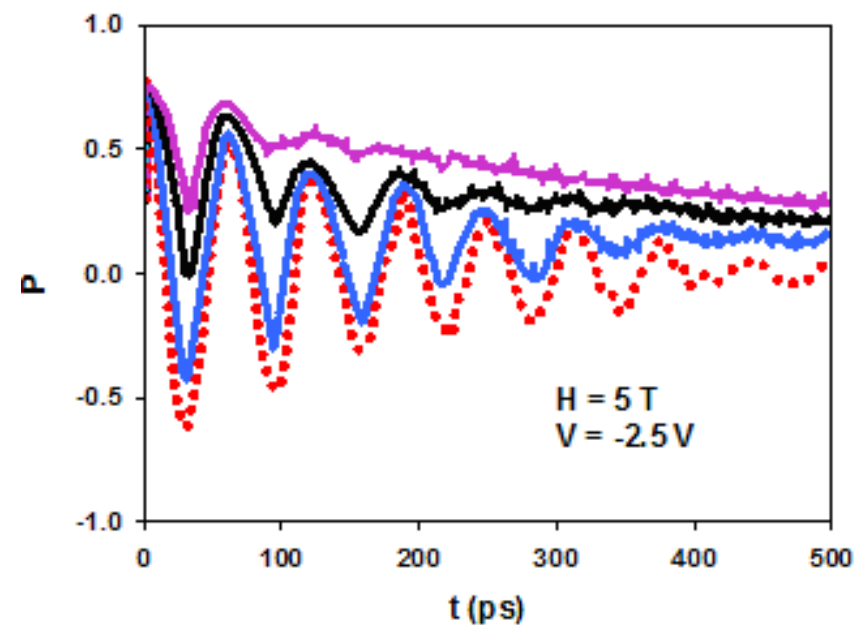

Fig. 7. The $P$ as a function of the angle between the magnetic field and the direction of observation (pink: $135^{\circ}$, black: $120^{\circ}$, blue: $105^{\circ}$ and red: $90^{\circ}$ ). 